\title{
O USO DE MAPAS DA REDE PESSOAL SOCIAL: ESTRATÉGIA DE PESQUISA E INTERVENÇÃO JUNTO A ADOLESCENTES EM SOFRIMENTO PSÍQUICO
}

\author{
Diene Monique Carlos' ${ }^{1}$, Isabela Martins Gabriel' ${ }^{1}$, Daniela Gonsalves Lopes ${ }^{1}$, Fernanda \\ Maranho Santos ${ }^{1}$, Ana Paula França de Oliveira ${ }^{1}$ e Luiza Cesar Riani Costa ${ }^{1}$ \\ ${ }^{1}$ Universidade Federal de São Carlos, Brasil. diene enf@hotmail.com isamartinsgabriel@hotmail.com; \\ d.aniela_lopes@yahoo.com.br; femaranho13@gmail.com ninhabtu34@gmail.com; luiza-riani@hotmail.com
}

\begin{abstract}
Resumo. Introdução: A adolescência constitui um período que, em comparação com outras faixas etárias, não apresenta grandes índices de adoecimento; entretanto as violências autoinfligidas têm ocupado destaque na sua morbimortalidade. Estratégias metodológicas para aproximação cuidadosa e ética destes fenômenos na adolescência, e com benefícios diretos aos sujeitos, têm sido recomendadas pela literatura. Objetivo: Analisar o uso do mapa da rede pessoal social como estratégia metodológica junto a adolescentes em sofrimento psíquico. Métodos: Estudo metodológico que relata a experiência do uso de mapas mínimos da rede pessoal social; realizado junto a adolescentes de escola pública de um município do interior de São Paulo, Brasil. Resultados: Em termos metodológicos e científicos, o uso dos mapas mostrou-se profícuo e coerente. $O$ uso de materiais a baixo custo e de fácil acesso e manuseio, permite a aplicação em diversos contextos e situações. Em termos éticos, permitiu o acesso cuidadoso a ausências experienciadas. Conclusões: $O$ uso desta estratégia foi importante tanto para 0 delineamento científico, acessando conhecimentos que dificilmente seriam trazidos por outros instrumentos de coleta, quanto para intervenção terapêutica delineada pela construção de dispositivos concretos de apoio.
\end{abstract}

Palavras-chave: Comportamento Autodestrutivo; Adolescente; Saúde Escolar; Rede Social; Pesquisa Qualitativa.

\section{THE USE OF INDIVIDUAL SOCIAL NETWORK MAPS: RESEARCH AND INTERVENTION STRATEGY TO ADOLESCENTS IN PSYCHOLOGICAL DISTRESS}

\begin{abstract}
Introduction: Adolescence is a period that, in comparison with other age groups, does not present high rates of illness; however, self-injured violence has been prominent in its morbidity and mortality. Methodological strategies for careful and ethical approach to these phenomena in adolescence, and with direct benefits to the individuals, have been recommended by the literature. Goals: Analyze the use of the individual social network map as a methodological strategy for adolescents in psychological distress. Methods: Methodological study that reports the experience of using minimal maps of the individual social network; conducted at a public school with adolescents in a country town of São Paulo, Brazil. Results: Considering the methodological and scientific issues, the use of maps proved to be meaningful and consistent. The use of low cost and easy access materials, allowed the application in different contexts and situations. By ethical terms, it allowed careful access to experienced absences. Conclusions: The use of this strategy was important both for the scientific design, accessing knowledge that would hardly be brought by other collection data instruments, and for therapeutic intervention designed by the construction of concrete support devices.
\end{abstract}

Keywords: Self-Injury Behavior; Adolescent; Scholar Health; Social Network; Qualitative Research.

\section{INTRODUÇÃO}

Apesar da diminuição do crescimento e aumento do envelhecimento populacional encontrado em países em desenvolvimento, como é o caso do Brasil, a representação de 
adolescentes e jovens na população geral é bastante significativa. Neste país, segundo o Instituto Brasileiro de Geografia e Estatística, em 2016 os adolescentes configuravam um total de 51.402.821 pessoas, cerca de 36,89\% da população (Brasil, 2017). O Ministério da Saúde seguindo as diretrizes da Organização Mundial da Saúde (OMS), utiliza o critério cronológico para definir a adolescência considerando dois períodos - o inicial entre 10 e 14 anos, e o final entre os 15 e os 19 anos. Já no Brasil, o Estatuto da Criança e do Adolescente (ECA), Lei 8.069 de 1990, define a adolescência como a faixa etária de 12 a 18 anos de idade, e, em casos excepcionais ao que se refere às medidas socioeducativas, quando disposto na lei, o estatuto é aplicável até os 21 anos de idade (Brasil, 2017).

Apesar das demarcações cronológicas, a adolescência é uma construção social relativamente recente. Trata-se de um período dinâmico, atravessado por marcas culturais e históricas, que não deve ser analisado apenas pelo viés cronológico ou biológico (Brasil, 2017). Tampouco deve ser compreendida apenas como período de preparação para a vida adulta ou como uma fase. A compreensão da adolescência, assim como a prevenção ou atenção a esta categoria social, deve ultrapassar concepções universalizantes e cristalizadas, pois apesar de ser possível fazer definições gerais, são sujeitos singulares constituídos de acordo com a história de vida e com o momento histórico e sociocultural em que vivem (Silva et al., 2014).

A adolescência constitui um período que, em comparação com outras faixas etárias, não apresenta grandes índices de adoecimento. Por conseguinte, segundo a OMS, a maior parte das mortes neste período são decorrentes de violências e acidentes, e podem ser evitadas. No mundo, a primeira causa de morte entre jovens de 15 a 29 anos é a violência interpessoal, e a segunda é a violência autoinfligida, constituída pelos comportamentos suicidas (World Health Organization, 2018). Já no Brasil, em 2015, o suicídio foi a quarta causa de morte nesta faixa etária, segundo dados do Ministério da Saúde (Brasil, 2017).

Os comportamentos suicidas são condenados por razões culturais ou religiosas em várias regiões do mundo; tendem a ser um ato secreto permeado por tabus, e pode ser não reconhecido ou classificado como tal e escondido em registros oficiais de morbimortalidade (World Health Organization, 2018). Na adolescência, é bastante associado a apoio parental e familiar fragilizado ou ausente (Macalli et al., 2018); história de violências intrafamiliares (Deville et al., 2020); baixo nível de apoio social em contexto escolar e comunitário (Miller, 
Esposito-Smythers \& Leichtweis, 2015); vivência de bullying (Lereya et al., 2015); uso abusivo de redes digitais (Marchant et al., 2017).

Estratégias metodológicas para aproximação cuidadosa e ética destes fenômenos na adolescência, e com benefícios diretos aos sujeitos, têm sido recomendadas pela literatura. Considerando a importância do contexto no delineamento das situações de sofrimento psíquico, em especial nos comportamentos suicidas pela atual prevalência na adolescência, e no empoderamento e protagonismo juvenil, traz-se a seguinte questão norteadora: Quais as contribuições do uso de mapas da rede pessoal social como estratégia metodológica de coleta de dados qualitativos junto a adolescentes em sofrimento psíquico?

\section{OBJETIVO}

O objetivo deste estudo foi analisar o uso do mapa da rede pessoal social como estratégia metodológica junto a adolescentes em sofrimento psíquico.

\section{METODOLOGIA}

Estudo metodológico que relata a experiência do uso de mapas mínimos da rede pessoal social como estratégia de coleta de dados qualitativos junto a adolescentes. A abordagem qualitativa é entendida como aquela destinada ao estudo de fenômenos e relações sociais, pela perspectiva de participantes diretamente envolvidos em determinada situação e contexto (Flick, 2009).

Os conceitos de rede e apoio social nortearam o percurso metodológico. Redes sociais são recursos construídos para circulação do apoio social (Andrade \& Vaitsmann, 2002). A literatura tem apresentado alguns tipos de apoio social, a saber emocional; informativo e instrumental. O apoio emocional diz respeito ao sentimento de pertencimento, estima ou valoração; demonstrações de carinho e amor. Apoio informativo é a provisão de fatos ou conselhos que podem ajudar uma pessoa a resolver problemas. Apoio instrumental consiste no oferecimento ou suplementação de assistência material para dúvidas ou problemas práticos (Thoits, 2011).

Foi realizado em um município do interior de São Paulo, Brasil, com 221.950 habitantes. O campo específico foi uma escola estadual de ensino fundamental e médio de um distrito do município. A escolha por este local se deu devido ao número elevado de situações de 
adoecimento psíquico referidas a serviços de saúde e ausência de ações de promoção de saúde mental locais.

As participantes foram incluídas mediante os critérios: (1) ser estudante matriculado na escola citada; (2) ter idade entre 10 e 19 anos; (3) ser indicado pela direção ou professores por estar em situação de sofrimento psíquico, em especial comportamentos suicidas. Foram excluídos os adolescentes afastados do convívio escolar por quaisquer motivos. Ressalta-se que a pesquisadora principal e sua orientadora participaram do convívio escolar por meio de um projeto de extensão.

A direção da escola indicou oito adolescentes do sexo feminino. Antes do início da coleta de dados, a direção realizou contato com os pais para anuência da participação das adolescentes. As pesquisadoras realizaram uma primeira conversa com as adolescentes, para convidá-las a participar do estudo, e todas aceitaram. A coleta de dados realizou-se entre os meses de agosto a novembro de 2019; o mapa foi realizado como estratégia complementar às entrevistas delineadas junto às adolescentes e para finalização. Pelas características deste estudo, não abordaremos os dados das entrevistas. O mesmo foi construído por uma das pesquisadoras, presente no convívio escolar das adolescentes, numa sala privativa na própria escola, sem a presença de outras pessoas. Os horários foram combinados com a equipe escolar de forma a evitar o prejuízo às atividades escolares.

O Mapa Mínimo da Rede Pessoal Social, proposto por Sluzki (1997), se constitui por um desenho representado por um círculo com quatro quadrantes principais: família, amizades, escola/trabalho, e relações comunitárias (religião, esporte, cinema, teatro, clubes, praças, entre outros). Além destes, há um quadrante que abarca a relação com serviços de saúde e de assistência social. Os quadrantes são permeados por outros dois círculos, que indicarão a intimidade e intensidade das relações, conforme representado na Figura 1:

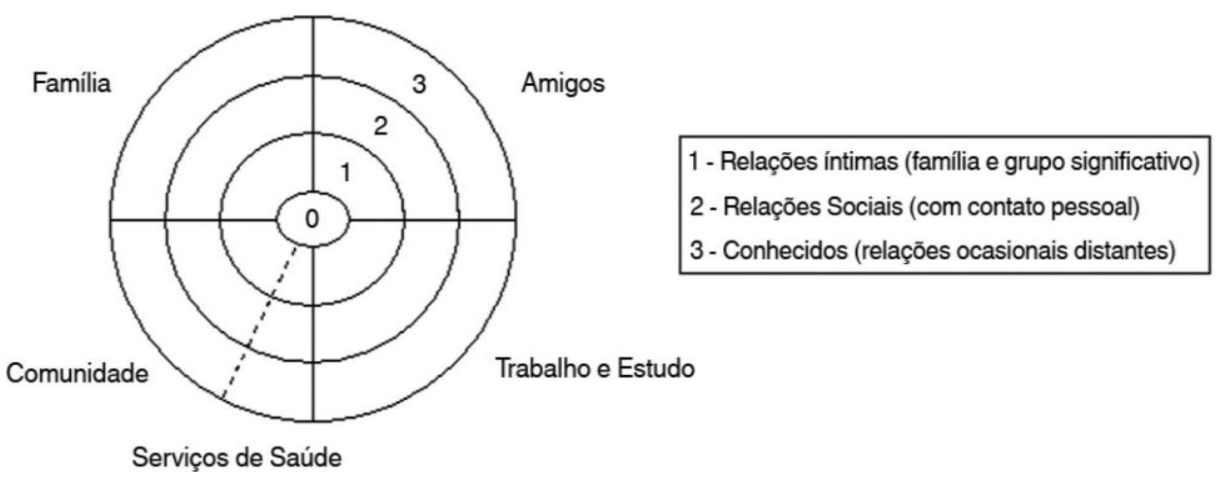

Figura 1. Modelo de Mapa Mínimo da Rede Pessoal Social (Sluzki, 1997) 
Além da representação da natureza das redes sociais, estes mapas permitem a compreensão da maneira que as relações se estabelecem. A qualidade dos vínculos é sinalizada por linhas que se referem aos vínculos significativos, fragilizados, rompidos ou inexistentes, descritos da seguinte maneira:

- Vínculos significativos - o traço utilizado é uma linha contínua, a qual pode ser desenhada com a cor verde ou preta;

- Vínculos fragilizados - são vínculos que se encontram tênues devido a diversos fatores. A representação gráfica se dá por meio do desenho de uma linha com a cor amarela ou por meio de uma linha preta pontilhada;

- Vínculos rompidos ou inexistentes -se avaliam aqueles vínculos que foram rompidos e lou inexistentes. Nesses casos, simboliza-se o vínculo utilizando uma linha quebrada ou na cor vermelha.

A avaliação do desenho dos mapas é realizada por meio dos seguintes critérios: tamanho (quantidade de vínculos institucionais/pessoais estabelecidos, sendo que a rede pode ser classificada como reduzida, mediana ou ampliada); densidade (qualidade dos vínculos observados no que tange às linhas do traçado - significativos, fragilizados, rompidos ou inexistentes); distribuição/composição (número de pessoas ou instituições situadas em cada quadrante, onde denotam-se lacunas e recursos existentes na rede); dispersão (distância geográfica entre membros e instituições); homogênea ou heterogênea (características dos membros e das instituições, no intuito de verificar a diversidade e as semelhanças que compõem a rede) (Sluzki, 1997).

Além destas características, reconhecem-se as funções da rede, ou seja, as potencialidades e vantagens para a implicação de uma sociedade mais articulada e comprometida, fortalecendo o trabalho comunitário pautado por parcerias solidárias (Sluzki, 1997). Tais funções são constituídas por: companhia social, apoio emocional, guia cognitivo e de conselhos, regulação e controle social, ajuda material e de serviços, e acesso a novos contatos. Esta última se refere ao envolvimento junto a redes cooperativas e solidárias, que possibilita a construção de novos vínculos pessoais, coletivos e institucionais, ampliando a rede pessoal dos participantes.

Os mapas foram impressos em papel A4 para uso junto às adolescentes. A construção dos mapas teve duração entre 30 a 35 minutos. Um diário de campo foi utilizado pela pesquisadora para relato do processo metodológico bem como de construção dos mapas 
após as conversas com as adolescentes. Todos os nomes presentes neste artigo foram fictícios, escolhidos pelas autoras. O anonimato das participantes foi preservado.

Os dados foram analisados mediante os critérios de avaliação dos mapas, citados anteriormente (Sluzki, 1997) e tipos de apoio social, reconhecendo as funções da rede (Thoits, 2011; Sluzki, 1997). Os mapas foram registrados em câmera fotográfica pela pesquisadora após concordância das participantes, sendo o mapa original construído mantido com as adolescentes. Algumas estratégias foram utilizadas para garantir maior confiabilidade interna e externa dos dados (Tong, Sainsbury \& Craig, 2007), a saber: devolutiva da dos mapas e da rede às adolescentes após construção, conforme critérios de analise citados; análise com pares; uso do diário de campo, garantindo maior transparência de todo o processo de pesquisa.

A pesquisa atendeu aos aspectos éticos envolvendo seres humanos, sendo aprovada pelo Comitê de Ética da Universidade Federal de São Carlos (CAAE: 17176219.6.0000.5504), e autorizada pela escola cenário da pesquisa.

Reitera-se que as informações foram coletadas apenas após assinatura do Termo de Consentimento Livre e Esclarecido pelos responsáveis e do Termo de Assentimento Livre e Esclarecido pelas adolescentes.

\section{RESULTADOS}

Inicialmente, foi solicitado às adolescentes que elencassem as pessoas e/ou serviços com que poderiam contar em momentos difíceis; elas tiveram dificuldades em lembrar e nomearam duas ou três pessoas. Ao apresentar a imagem do mapa e verem seus quadrantes, conseguiram inserir outras pessoas e instituições.

As redes se apresentaram reduzidas a medianas, com grandes variações entre as adolescentes. Mostraram-se pouco densas visto a existência de vínculos frágeis ou rompidos, sendo os recursos existentes concentrados em famílias e amigos. Delineou-se portanto uma rede homogênea. Perceberam-se lacunas importantes na rede essencialmente nos campos comunitário e estudo. Um dos mapas é representado na Figura 2: 


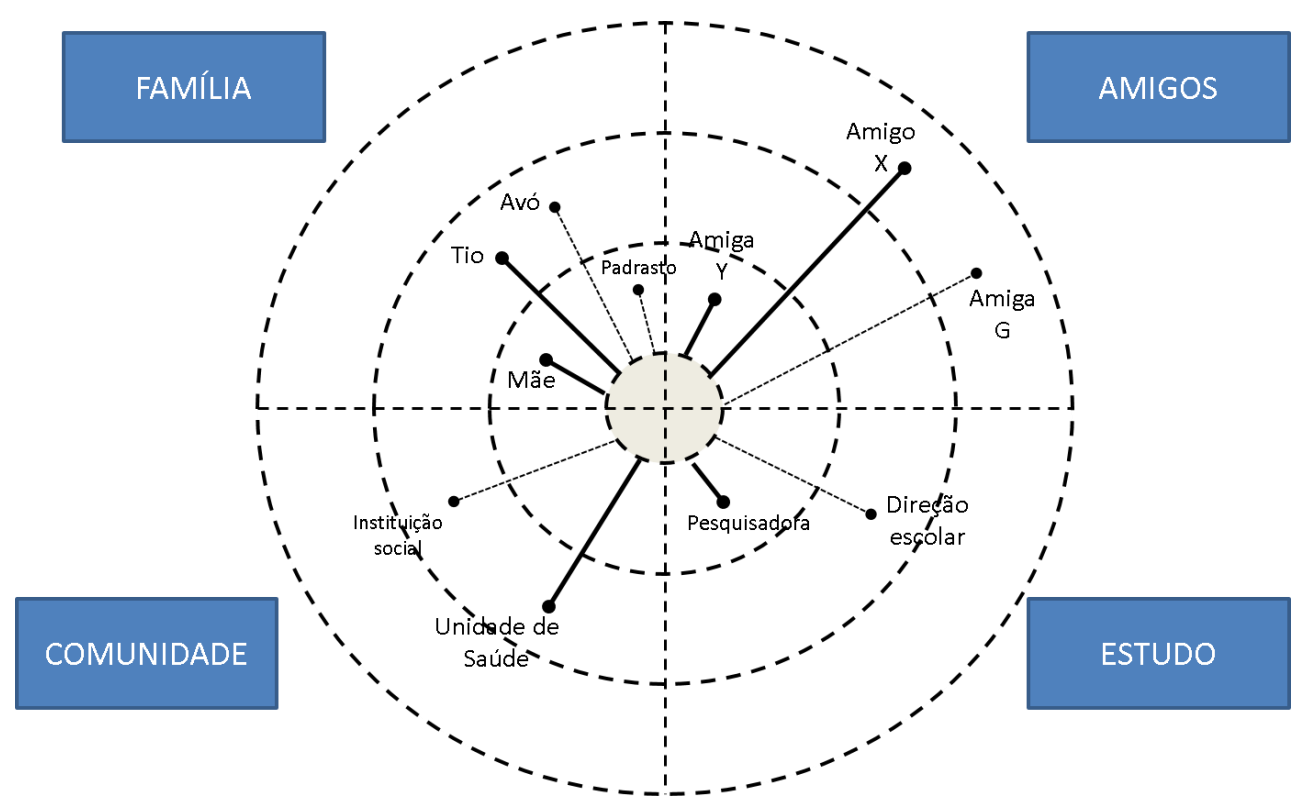

Figura 2. Mapa Mínimo da Rede Pessoal Social da adolescente Karina

Três adolescentes citaram professores como vínculos significativos. Duas adolescentes relataram que a pesquisadora seria um vinculo significativo. Os serviços de saúde foram vínculos significativamente apenas por uma participante.

Ademais, revelou-se pouca circulação de apoio social, seja afetivo, informativo ou instrumental. $O$ apoio afetivo foi oriundo essencialmente de familiares e amigos. Uma adolescente pontuou o apoio afetivo e informativo a coordenação escolar. Os apoios informativo e instrumental, tão necessários na adolescência visto construção da autonomia, não foram relatados pelas adolescentes.

As adolescentes se mostraram abertas ao conhecimento de novos serviços que poderiam exercer papel protetivo, sendo estes acrescentados ao mapa em azul. Estes serviços foram sinalizados pela pesquisadora, a saber Centro de Valorização à Vida; Centro de Referência em Assistência Social; Unidade de Saúde da Família.

O mapa teve uma boa aceitação, e as adolescentes compreenderam rapidamente como fazer. Houve uma pequena confusão entre a diferença das cores, significando a qualidade das relações, e da distância do centro, que significava a intimidade das relações. Mesmo após explicação, as adolescentes continuaram associando tais achados. 
As adolescentes referiram ser uma "atividade leve"; na finalização, mostraram-se mais confiantes em perceber a concretude da rede e optaram por manter os mapas consigo.

Em termos metodológicos e científicos, o uso dos mapas mostrou-se profícuo e coerente. $O$ uso de materiais a baixo custo e de fácil acesso e manuseio, permite a aplicação em diversos contextos e situações. Os mapas são de fácil compreensão, sendo adequados para diferentes faixas etárias, desenvolvimentos cognitivos e populações. Algumas adaptações, como ocorridas neste estudo, são viáveis desde que bem reportadas.

Como técnica de coleta de dados, permite ser complementada ou complementar a muitas outras, como photovoice, entrevistas, grupos focais. Também pode ser trabalhada em estudos de métodos mistos. A análise de dados pode ser associada a outras estratégias, bem com articulada a iferentes referenciais teóricos.

Em termos éticos, permite o acesso cuidadoso a ausências e lacunas experienciadas. Ademais, traz um benefício imediato a quem participa dele - a possibilidade terapêutica de acessar sua rede, perceber as fragilidades e potencialidades e buscar maneiras de novos contatos para superar as dificuldades. O/a pesquisador/a necessita estar preparado e capacitado para esta aproximação e manejo da situação, garantindo melhor aproveitamento da estratégia.

\section{DISCUSSÃO}

Os mapas mínimos da rede pessoal social se apresentaram como importantes e coerentes estratégias de coleta de dados qualitativos. A partir do desenho metodológico, podem apoiar outras técnicas, serem disparadores ou estratégias principais. Ademais, se mostram adaptáveis a vários objetos de estudo e populações, bem como se fazem coerentes a variados referenciais teóricos.

Neste estudo, optou-se pela abordagem a situações de sofrimento psíquico evidenciadas por comportamentos de violências autoinfligidas na adolescência. A adolescência é uma categoria social, marcada por um processo de desenvolvimento que sofre impacto direto do contexto histórico, político e social em que está inserida (Brasil, 2017). Ainda é compreendida pela sociedade em geral, incluindo profissionais da educação e da saúde, como uma fase passageira e com olhar determinista e generalizado (Brasil, 2017). 
Desvelou-se no presente estudo a ausência de ações intersetoriais que promovam o protagonismo e autonomia destes sujeitos, em especial a educação e saúde.

Os adolescentes têm se destacado nas estatísticas de violências autoinfligidas; a literatura reforça que o fenômeno da autolesão trata-se de uma expressão particular de angustia emocional com importante relutância em buscar ajuda (Doyle, Sheridan, \& Treacy, 2017). Estudo que analisou os temas relacionados ao fenômeno em postagens de blogs, encontrou que o comportamento pró-suicida foi apresentado como a única forma para cessar um sofrimento; nestes termos, desamparo e sofrimento continuo foram temas principais abordados (Costa, Silva \& Vedana, 2019). Percebe-se a fragilidade da rede pessoal das adolescentes, em que não percebem outra possibilidade de enfrentar e lidar com o sofrimento que não passe pela autolesão ou suicídio.

Em estudo que buscou identificar estratégias de prevenção a comportamentos suicidas na Inglaterra, sugeriram que ter um suporte social capaz de escutar e acolher é um importante meio de prevenção e de demonstrar apoio para adolescentes que sentem vontade de se autolesionar (Fortune, Sinclair, \& Hawton, 2008). Neste mesmo estudo os adolescentes apontaram que sentem frequentemente desejo de mais amor, atenção, tempo, apoio e cuidado por parte das famílias, que devem mostrar mais suporte e compreensão a respeito das mudanças enfrentadas na adolescência. Também foi mencionado que as amizades e relações entre pares são fatores de proteção. Outro estudo realizado na Bélgica e Holanda apontou que apoio familiar oferece ao adolescente oportunidade de enfrentar sentimentos depressivos adequadamente, no lugar de recorrer a violências autoinfligidas (Claes et al., 2015). Acessar lacunas e fragilidades das redes e do consequente apoio social oriundo delas pode ser o primeiro passo para a construção e estratégias promotoras de saúde.

Estudo que documentou a atual diversidade e complexidade de famílias de adolescentes para repensar futuras teorias parentais e estudos, recomenda o uso de mapas da rede de apoio junto a adolescentes (Pearce et al., 2018). Estes mapas, tendo o adolescente como centro da rede, permitem uma visão das dimensões-chave de estruturas e processos familiares (Pearce et al., 2018). Ademais, a literatura tem reforçado a importância do protagonismo e do ouvir as vozes dos adolescentes, colocando-os efetivamente como centro no cuidado. O envolvimento da pessoa nas decisões do cuidado contribui para satisfação de adolescentes e jovens (Mitten et al., 2016; Doyle, Sheridan, \& Treacy, 2017). 
Métodos qualitativos de pesquisa nos permitem melhor compreender as experiências das pessoas, explorar como decisões são tomadas e prover ideias com detalhes de como o cuidado pode ser qualificado. Para tal, estes dados precisam ser holísticos, ricos e com nuances (Barrett \& Twycross, 2018). Neste sentido, pesquisadores têm utilizado a abordagem das redes e mapas sociais para compreender fenômenos complexos, como o apoio na gravidez na adolescência (Braga et al., 2014); violência contra a mulher (Netto et al., 2017) e contra crianças e adolescentes (Carlos et al., 2019).

Recente discussão sobre o uso de redes sociais trouxe a necessidade de alguns cuidados na interpretação dos resultados destes estudos (Parnell \& Robinson, 2018). Um deles é a não generalização, que deve ser tomada cuidadosamente visto que toda rede de apoio é única. Nos estudos qualitativos, tal aspecto já é considerado; no momento de apresentação dos resultados, os mesmos podem ser tomados como complementares entre os participantes. Apesar de abordarmos o uso desta estratégia de coleta em estudos qualitativos, a mesma pode ser utilizada em estudos mistos (Parnell \& Robinson, 2018). Também se faz importante e demonstra maior validade e confiabilidade científicas, a devolução dos dados aos participantes; tal aspecto pode trazer contribuições tanto à pessoa quanto à pesquisa, como realizado no presente estudo.

O estudo citado também sinaliza o cuidado ético, visto que a confidencialidade deve ser mantida; pelas especificidades, o completo anonimato pode ser difícil, tendo os pesquisadores este desafio de buscar estratégias para tal. Este aspecto se estende a pessoas ou grupos, visto que as redes podem ser representativas de um coletivo, geralmente comunidades vulneráveis, que devem ser protegidas e ter um olhar sensível a esta questão (Parnell \& Robinson, 2018).

A análise a partir de mapas de redes permitem a compreensão de um mundo heterogêneo construído por indivíduos autônomos que co-criam ambientes que produzem resultados em saúde. Estes resultados são frequentemente frutos da influência cultural de relações interpessoais e comunitárias, delineadas pela rede. Destaca-se que os mapas da rede capturam processos sociais que variam culturalmente, permitindo a profissionais de saúde que ofereçam um cuidado mais efetivo (Parnell \& Robinson, 2018). 


\section{CONCLUSÕES}

Os resultados deste estudo permitiram acessar elementos que demonstram a contribuição do uso de mapas da rede pessoal como estratégia de coleta de dados qualitativos, evidenciados por meio de estudo com adolescentes em sofrimento psíquico. O uso desta estratégia foi importante tanto para o delineamento científico, acessando conhecimentos que dificilmente seriam trazidos por outros instrumentos de coleta, quanto para intervenção terapêutica delineada pela construção de dispositivos concretos de apoio. A visualização gráfica da rede permite uma "memória" de possibilidades de busca de ajuda, bem como trabalha na lógica salutogênica.

O estudo traz contribuições para a investigação, assistência e formação em saúde. Para a investigação, ilustra técnica de coleta de dados adaptável a diferentes objetos de estudo, referenciais, populações e estratégias metodológicas. Ademais, se articula às atuais demandas éticas em constructos qualitativos. Quanto à assistência, proporciona implicações para intervenções junto a adolescentes em sofrimento psíquico, com foco em sua autonomia e protagonismo. Para a formação, pode ser incluído como parte curricular do ensino em saúde e educação, pela facilidade de compreensão e acesso seja na pesquisa ou prática.

As limitações do presente estudo se relacionam à particularidade do objeto. Entretanto, traz importantes contribuições a desenhos metodológicos qualitativos em especial delineado junto a populações e grupos vulneráveis. Estudos que abordem tal estratégia a estudos de métodos mistos são recomendados.

Agradecimentos. À Fundação de Amparo à Pesquisa do Estado de São Paulo pela bolsa de Iniciação Científica (Processo 2019/09333-9).

\section{REFERÊNCIAS}

Andrade G.R.B., \& Vaitsman, J. (2002). Apoio social e redes: conectando solidariedade e saúde. Ciência \& Saúde Coletiva, 7(4):925-934.

Barrett D., \& Twycross A. (2018). Data collection in qualitative research. Evidence-Based Nursing, 21, 63-64.

Braga, I.F., Oliveira, W.A., Spanó, A.M.N., Nunes, M.R., \& Silva, M.A.I. (2014). Percepções de adolescentes sobre o apoio social na maternidade no contexto da atenção primária. Escola Anna Nery, 18(3), 448455. doi: 10.5935/1414-8145.20140064

Brasil. Ministério da Saúde. (2017). Proteger e Cuidar da Saúde de Adolescentes na Atenção Básica. Brasília (DF): Ed. Ministério da Saúde.

Carlos D.M., da Silva L.M.P., Beserra M.A., Aragão A.S., Gregory A., \& Ferriani M.G.C. (2019). Social support network of family members of abused children and adolescents: Perspectives and possibilities. Journal of Clinical Nursing, 28(5-6), 814-27. doi: 10.1111/jocn.14665. 
Claes L., Luyckx K., Baetens I., Van de Ven M., \&Witteman C. (2015). Bullying and victimization, depressive mood, and non-suicidal self-injury in adolescents: The moderating role of parental support. Journal of child and family studies, 24(11), 3363-3371.

Costa J.S., Silva A.C., \& Vedana K.G.G. (2019). Postagens sobre autolesão não suicida na internet. Adolescência \& Saude, 16(1), 7-12.

Deville D.C., Whalen D., Breslin F.J., Morris A.S., Khalsa S.S., Paulus M.P. et al. (2020). Prevalence and FamilyRelated Factors Associated With Suicidal Ideation, Suicide Attempts, and Self-injury in Children Aged 9 to 10 Years. JAMA Netw Open, 3(2), e1920956. doi: 10.1001/jamanetworkopen.2019.20956

Doyle L., Sheridan A., \& Treacy M.P. (2017). Motivations for adolescent self-harm and the implications for mental health nurses. Journal of Psychiatric Mental Health Nursing, 24(2-3), 134-142. doi: 10.1111/jpm.12360.

Flick U. (2009). An introduction to qualitative research. 5th ed. Thousand Oaks, CA: Sage.

Fortune S., Sinclair J., \& Hawton K. (2008). Adolescents' views on preventing self-harm. Social Psychiatry and Psychiatric Epidemiology, 43(2),96-104.

Lereya S.T.E., Copeland W., Costello J. \& Wolke D. (2015). Adult mental health consequences of peer bullying and maltreatment in childhood: two cohorts in two countries. The Lancet Psychiatry, 2(6), 524-31. doi: 10.1016/S2215-0366(15)00165-0.

Macalli M., Tournier M., Galéra C., Montagni I., Soumare A., Côté S.M. et al. (2018). Perceived parental support in childhood and adolescence and suicidal ideation in young adults: a cross-sectional analysis of the iShare study. BMC Psychiatry, 18(1),373. doi: 10.1186/s12888-018-1957-7.

Marchant A., Hawton K., Stewart A., Montgomery P., Singaravelu V., Lloyd K. et al. (2017). A sistematic review of the relationship between internet use, self-harm and suicidal behavior in Young people: the good, the bad and the unknown. PLoS ONE, 13(3), e0193937. doi: 10.1371/journal.pone.0181722

Miller A.B., Esposito-Smythers C., \& Leichtweis R.N. (2015). Role of social support in adolescent suicidal ideation and suicide attempts. Journal of Adolescent Health, 56(3), 286-292. doi: 10.1016/j.jadohealth.2014.10.265

Mitten N., Preyde M., Lewis S., Vanderkooy J., \& Heintzman J. (2016). The perceptions of adolescents who selfharm on stigma and care following inpatient psychiatric treatment. Social Work in Mental Health, 14(1), 121. doi: $10.1080 / 15332985.2015 .1080783$

Netto, L.A., Moura, M.A.V., Araujo, C.L.F., Souza, M.H. N., \& Silva, G.F. (2017). As redes sociais de apoio às mulheres em situação de violência pelo parceiro íntimo. Texto \& Contexto - Enfermagem, 26(2), e07120015. Doi: 10.1590/0104-07072017007120015

Parnell, J. M., \& Robinson, J. C. (2018). Social network analysis: Presenting an underused method for nursing research. Journal of advanced nursing, 74(6), 1310-1318. https://doi.org/10.1111/jan.13541

Pearce L.D., Hayward G.M., Chassin L., \& Curran P.J.(2018) The increasing diversity and complexity of family structures for adolescents. Journal of Research on Adolescence, 28(3), 591-608. doi: 10.1111/jora.12391.

Silva M.A.I, de Mello F.C.M., de Mello D.F., Ferriani M.G.C., Sampaio J.M.C. \& de Oliveira W.A. (2014). Vulnerability in adolescent health: contemporary issues]. Ciência \& saúde coletiva, 19(2),619-27. doi: 10.1590/1413-81232014192.22312012. Portuguese.

Sluzki, C.E. (1997). A rede social na prática sistêmica: alternativas terapêuticas. São Paulo: Casa do Psicólogo.

Tong A., Sainsbury P., Craig J. (2007). Consolidated criteria for reporting qualitative research (COREQ): a 32item checklist for interviews and focus groups. International Journal for Quality in Health Care, 19(6): 349357. Doi:10.1093/intqhc/mzm042

Thoits P. A. (2011). Perceived Social Support and the Voluntary, Mixed, or Pressured Use of Mental Health Services. Society and Mental Health, 1(1), 4-19. https://doi.org/10.1177/2156869310392793

World Health Organization. (2020). Suicide factsheet. Acesso em 21 Jan 2020. Disponível em: https://www.who.int/violence_injury_prevention/violence/world_report/factsheets/en/selfdirectedviolfacts.pdf?ua=1 